'Grupo Correos-Valencia. IINSS-Madrid

${ }^{3}$ Servicio de Prevención

Mancomunado MAPFRE. Valencia.

${ }^{4}$ Grupo Correos-Albacete.

${ }^{5}$ Servicio de Prevención

de GESMA. Palma de Mallorca.

${ }^{6}$ Universidad Illes Balears. aDoctor (a) en Medicina del Trabajo.

bLicenciada en Medicina del Trabajo.

Este estudio no ha contado con apoyo financiero ni en el diseño del estudio; en la recolección, análisis o interpretación de los datos; en la preparación, revisión o aprobación del manuscrito.

Recibido el 23 de junio de 2012, aceptado el $10 \mathrm{de}$ diciembre de 2012

Correspondencia a: Dra. Ma Teófila Vicente Herrero.

Especialista en Medicina del Trabajo.

UBS- Grupo Correos. Valencia. España.

Plaza del Ayuntamiento, $24-2^{\circ}$

46002 Valencia. España. Teléfono: 963102752 Fax: 963940500

E-mail: MTVH@ono.com/ correoteo@gmail.com/ grupo.gimt@gmail.com

\section{La incapacidad temporal en España por algunas enfermedades psiquiátricas. (Trastornos esquizofrénicos, trastorno bipolar y trastornos ansiosos, depresivos y adaptativos)}

\author{
$\mathrm{M}^{\mathrm{a}}$ TEÓFILA VICENTE-HERRERO ${ }^{1, \mathrm{a}}, \mathrm{M}^{\mathrm{a}}$ JESÚS TERRADILLOS GARCÍA ${ }^{2, \mathrm{~b}}$, \\ LUISA M. CAPDEVILA GARCÍA ${ }^{3, a}$, \\ $M^{\mathrm{a}}$ VICTORIA RAMÍREZ IÑIGUEZ DE LA TORRE ${ }^{4, \mathrm{~b}}$, \\ ÁNGEL ARTURO LÓPEZ-GONZÁLEZ ${ }^{5,6, a}$
}

\section{Sick leaves caused by psychiatric diseases in Spain during 2011}

Background: Psychiatric and mood disorders may hamper the efficiency of workers and cause absenteeism. Material and Methods: Revision of sick leaves related to schizophrenia, bipolar diseases, anxiety, depression and adaptive disorders that were granted in Spain in the period January-December 2011. The mean number of days off work for each disease and the costs per each labor day lost were calculated. Results: The total number of labor days lost for schizophrenia, bipolar diseases and mood disorders were 72,831; 899, 107 and 3,666,055 days, respectively. The mean duration of sick leaves for the same diagnoses were 84; 100 and 64 days/year respectively. The total indirect costs of sick leaves for psychiatric diseases during 2011 were $€ 100$ million approximately, corresponding to $4 \%$ of costs for all diseases. Conclusions: The high costs of absenteeism derived from psychiatric diseases should prompt measures to promote a better adaptation to work environments of people suffering these ailments.

(Rev Med Chile 2013; 141: 248-254).

Key words: Costs and costs analysis; Mental disorders; Occupational health; Sick leave.
L

as enfermedades psiquiátricas constituyen la segunda causa de incapacidad temporal en España, después de los trastornos músculoesqueléticos. En la sanidad pública y en el mundo del trabajo, adquieren especial significación al tratar de conjugar las opciones laborales del trabajador afectado que faciliten su desarrollo profesional y personal, con políticas de gestión eficaces.

Los trastornos mentales pueden generar consecuencias en la conducta de los enfermos y repercutir de forma negativa en su actividad laboral, originando incapacidad temporal (IT) o permanente (IP) con los efectos correspondientes ${ }^{2}$.
Según estimaciones del Banco Mundial y la OMS, al menos el 25\% de la "carga global de las enfermedades" se atribuye en la actualidad a los trastornos mentales y la evaluación en pérdidas se realiza años de vida perdida ajustados por incapacidad (DALY), la esquizofrenia, el trastorno afectivo bipolar y la depresión mayor suman el $10,8 \%$ del total $^{3}$.

La IT por contingencia común se considera en todos los países un importante problema de salud pública con repercusiones socioeconómicas ${ }^{4}$ y su gestión debe considerarse una prescripción médica, cuya evaluación y control afecta de forma directa 
al sistema sanitario como mejor conocedor de su origen, evolución y de todos sus factores asociados.

$\mathrm{Al}$ intentar realizar una aproximación somera al coste de estas patologías en base a la IT, se parte ya de inicio del sesgo de incluir en él únicamente trabajadores en activo y de no poder hacer un cálculo exacto del coste de un día de jornada laboral perdida por IT, pues dependerá de diferentes factores: el sector profesional al que pertenece el trabajador afectado, el puesto de trabajo desempeñado, el grupo de cotización en el que se encuentra incluido, a quién corresponde el pago de los tres primeros días de baja (a la empresa o al propio trabajador) y un sin número de cuestiones paralelas que resultan imposibles de contabilizar en un trabajo de estas características.

La prestación económica por IT en España, trata de cubrir la falta de ingresos que se produce cuando el trabajador, debido a una enfermedad o accidente, está imposibilitado temporalmente para trabajar y precisa asistencia sanitaria de la Seguridad Social. Esta prestación económica está incluida dentro de la acción protectora del Régimen General y de los regímenes especiales de la Seguridad Social.

Existen diferentes variables implicadas en el reconocimiento del derecho, la gestión y el pago de la prestación por IT; así, atendiendo al organismo pagador, la prestación puede correr a cargo de: Instituto Nacional de la Seguridad Social (INSS), Instituto Social de la Marina (ISM), Mutuas de Accidentes de Trabajo y Enfermedades Profesionales de la Seguridad Social (MATEPSS), empresas colaboradoras autoaseguradoras, Funcionarios Civiles del Estado (MUFACE), Funcionarios de las Administraciones Locales, Funcionarios de Justicia (MUGEJU); por otro lado, la modalidad de pago de la prestación puede ser: pago directo o pago delegado; y según el Régimen de Seguridad Social en que se incluye al trabajador: General, Especial de Trabajadores Autónomos, Especial de la Minería del Carbón, Especial de Trabajadores del Mar; la contingencia de la IT puede ser: común o profesional. Independientemente de ello cabe reseñar las diferencias en el concepto de incapacidad y discapacidad en España y el diferente proceso de valoración de ambas situaciones ${ }^{5}$.

Son múltiples las variables a considerar y la imposibilidad de abarcar tan sólo una mínima parte de ellas, hace que se dificulte la obtención de un cálculo real de coste/día por IT.
Revisemos unos datos someros de cada una de estas enfermedades que sirva de base para destacar su relevancia:

Los pacientes con esquizofrenia constituyen el $10 \%$ de las personas enfermas con IP y sólo $60-70 \%$ logran incorporarse al mundo laboral. La prevalencia varía según los distintos estudios, desde 4-7 por cada 1.000 personas, dependiendo del tipo de estimación que se realice. Su inicio puede ser a cualquier edad, la mayoría entre los 15 y los 54 años, con máximo entre los 20 y 26 años en los varones. En las mujeres se inicia 5 años después que en los hombres, y luego por encima de los 60 años. En ambos casos, el inicio coincide con la edad laboral activa y dificultan o impiden su desarrollo óptimo ${ }^{6}$.

El trastorno bipolar I, tiene una prevalencia del $0,8 \%(0,4$ a $1,6 \%)$ y el trastorno bipolar II, $0,5 \%$ de la población adulta ${ }^{7}$. Las prevalencias varían desde $2,6 \%{ }^{8}$ hasta $6,4 \%{ }^{9}$, según cual sea el estudio consultado. El mayor gasto de esta enfermedad es el asociado a los costes indirectos, ligados a pérdida de productividad, absentismo e IT $^{10}$.

En los trastornos de ansiedad, depresión y trastornos adaptativos, los estudios de prevalencia realizados recientemente, en 2010 en Irlanda, destacan la depresión como muy común entre la población estudiada, si bien los trastornos de ansiedad representan la más común de las enfermedades psiquiátricas y co-morbilidades en la población general ${ }^{11}$.

Reseñar finalmente el estudio realizado en Suecia relativo al coste global de las patologías mentales y cerebrales en Europa (coste per capita), que de forma global supondrían de media $€ 1.550$, variando este promedio según cada país ${ }^{12}$.

Los criterios diagnósticos de todas estas patologías se encuentran recogidos por la American Psychiatric Association-DSM-5 Development ${ }^{13}$.

\section{Objetivos}

1. Determinar el número de procesos de IT habidos en España durante el año 2011 en estas patologías incluyendo todos los códigos CIE 9 relacionados con ellas.

2. Determinar el total días perdidos por IT por estos procesos.

3. Determinar la duración media de cada proceso.

4. Cuantificar la repercusión de estas patologías en el global de las IT contabilizadas por todas las patologías en España en 2011. 
5. Realizar una aproximación referencial al coste derivado de IT en base al coste/día establecido en el SMI en España durante el año 2011.

\section{Métodos}

Se realiza una revisión de datos oficiales de códigos CIE-9 MC relacionados con las patologías referidas en España durante el período enerodiciembre de 2011 cuantificando el número de procesos, la duración de cada uno, su promedio de duración y las diferencias habidas por meses a lo largo del año.

Se consideran los siguientes códigos CIE-9 en cada una de las patologías:

\section{Trastornos esquizofrénicos}

295, 295.0, 295.00, 295.02, 295.1, 295.14, 295.2, 295.20, 295.3, 295.30, 295.31, 295.32, 295.33, 295.34, 295.35, 295.4, 295.40, 295.42, 295.5, 295.50, 295.52, 295.54, 295.6, 295.60, 295.7, 295.70, 295.72, 295.74, 295.75, 295.8, 295.82, 295.83, 295.84, 295.9, 295.90, 295.92, 295.93.

\section{Trastorno bipolar}

296, 296.0, 296.00, 296.02, 296.06, 296.1, 296.11, 296.12, 296.13, 296.14, 296.2, 296.20, 296.21, 296.22, 296.23, 296.24, 296.25, 296.3, 296.30, 296.31, 296.32, 296.33, 296.34, 296,35, 296.4, 296.40, 296.41, 296.42, 296.43, 296.44, $296.45,296.5,296.50,296.51,296.52,296.53$, 296.54, 296.55, 296.56, 296.6, 296.60, 296.61, 296.63, 296.64, 296.65, 296.7, 296.8, 296.80, 296.81, 296.82, 296.83, 296.9, 296.90, 296.99.

\section{Trastorno ansioso, depresivo, síndromes adaptativos}

$300,300.0,300.00,300.01,300.02,300.09$, 309.0, 309.1, 309.21, 309.24, 309.28, 311 у 313.

Para establecer comparativa de estas patologías respecto al global, se toma como base el total de enfermedades con IT recogidas por el INSS durante el 2011 y para el cálculo del gasto y referencia respecto al total, se considera el gasto global del INSS por IT en todas las patologías durante el 2011, excluidas empresas colaboradoras (se incluye pago directo y pago delegado).

Se realiza un cálculo de costes aproximativo y de mínimos partiendo del coste/día de trabajo de menor cuantía, que es el que hace referencia al SMI en España durante el 2011 según los da- tos oficiales publicados y teniendo en cuenta los sesgos que suponen las diferencias en las bases de cotización de los distintos tipos de trabajadores y que sólo incluye el gasto del Instituto Nacional de la Seguridad Social (INSS), no incluimos los días de baja que paga el empresario (los primeros 15 días de cada proceso). Se incluyen sólo los procesos de trabajadores que tienen las contingencias con el Instituto Nacional de la Seguridad Social, puesto que los que están con Mutuas no están incluidos.

El SMI estipulado en España para el 2011, que quedó fijado en $641,40 €$ mensuales, $21,38 € /$ día y 8.979,60€ anuales. Cualquier aproximación a cifras reales, deberá superar las del SMI, que fija la cuantía retributiva mínima que percibirá el trabajador referida a la jornada legal de trabajo, sin distinción de sexo o edad de los trabajadores, sean fijos, eventuales o temporeros. El valor que toma el SMI se fija cada año por el Gobierno, mediante Real Decreto y para la determinación del mismo se tienen en cuenta factores como el IPC, la productividad media nacional alcanzada o el incremento de la participación del trabajo en la renta nacional.

\section{Resultados}

Los resultados individuales en cada una de las patologías o grupos de patologías para 2011, se muestran en las Tablas 1, 2 y 3.

\section{Número de procesos de IT por estas patologías}

- Esquizofrenia y códigos CIE 9 asociados: 868 procesos.

- Trastorno bipolar y códigos CIE 9 asociados: 8.983 procesos.

- Trastorno de ansiedad, depresivos y adaptativos y códigos CIE 9 asociados: 56.795 procesos.

\section{Jornadas perdidas por IT en estas patologías}

- En esquizofrenia y códigos CIE 9 asociados: 72.831 días.

- Trastorno bipolar y códigos CIE 9 asociados: 899.107 días.

- Trastorno de ansiedad, depresivos y adaptativos y códigos CIE 9 asociados: 3.666.055 días.

\section{Duración media de los procesos IT en estas patologías:}

- Esquizofrenia y códigos CIE 9 asociados: 83,9 días/año de promedio. 
Tabla 1. Datos globales de trastornos esquizofrénicos-IT 2011

\begin{tabular}{|lccc|}
\hline Mes de 2011 & $\begin{array}{c}\text { Duración } \\
\text { media }\end{array}$ & $\begin{array}{c}\text { Número de } \\
\text { procesos }\end{array}$ & $\begin{array}{c}\text { Número } \\
\text { de días }\end{array}$ \\
\hline Enero & 102,4 & 60 & 6.143 \\
\hline Febrero & 98,8 & 85 & 8.397 \\
\hline Marzo & 91,5 & 76 & 6.957 \\
\hline Abril & 74,9 & 70 & 5.242 \\
\hline Mayo & 86,3 & 77 & 6.648 \\
\hline Junio & 83,3 & 102 & 8.499 \\
\hline Julio & 83,3 & 78 & 6.500 \\
\hline Agosto & 76 & 55 & 4179 \\
\hline Septiembre & 69,2 & 66 & 4.565 \\
\hline Octubre & 104,2 & 66 & 6.880 \\
\hline Noviembre & 72,4 & 83 & 6.010 \\
\hline Diciembre & 56,2 & 50 & 2.811 \\
\hline Total & 83,9 & 868 & 72.831 \\
\hline
\end{tabular}

Tabla 3. Datos globales de trastorno ansiosos, depresivos y adaptativo -IT 2011

\begin{tabular}{|lccc|}
\hline $\begin{array}{l}\text { Mes año } \\
\mathbf{2 0 1 1}\end{array}$ & $\begin{array}{c}\text { Duración } \\
\text { media }\end{array}$ & $\begin{array}{c}\text { Número de } \\
\text { procesos }\end{array}$ & $\begin{array}{c}\text { Número } \\
\text { de días }\end{array}$ \\
\hline Enero & 72,8 & 3.860 & 280.893 \\
\hline Febrero & 65,6 & 4.838 & 317.293 \\
\hline Marzo & 62,1 & 5.900 & 366.650 \\
\hline Abril & 60,1 & 5.217 & 313.455 \\
\hline Mayo & 62,5 & 5.280 & 330.152 \\
\hline Junio & 59,1 & 5.738 & 339.198 \\
\hline Julio & 65,9 & 5.196 & 342.670 \\
\hline Agosto & 64,3 & 3.536 & 227.205 \\
\hline Septiembre & 79,0 & 4.334 & 342.333 \\
\hline Octubre & 66,8 & 4.361 & 291.290 \\
\hline Noviembre & 62,6 & 4.648 & 291.042 \\
\hline Diciembre & 57,6 & 3.887 & 223.874 \\
\hline Total & 64,5 & 56.795 & 3.666 .055 \\
\hline
\end{tabular}

\begin{tabular}{|lccc|}
\hline $\begin{array}{l}\text { Mes año } \\
\mathbf{2 0 1 1}\end{array}$ & $\begin{array}{c}\text { Duración } \\
\text { media }\end{array}$ & $\begin{array}{c}\text { Número de } \\
\text { procesos }\end{array}$ & $\begin{array}{c}\text { Número } \\
\text { de días }\end{array}$ \\
\hline Enero & 96,3 & 578 & 55.672 \\
\hline Febrero & 115,1 & 733 & 84.401 \\
\hline Marzo & 101,4 & 836 & 84.753 \\
\hline Abril & 108,8 & 723 & 78.677 \\
\hline Mayo & 100,3 & 769 & 77.127 \\
\hline Junio & 96,6 & 832 & 80.345 \\
\hline Julio & 94,6 & 803 & 75.931 \\
\hline Agosto & 95 & 624 & 59.301 \\
\hline Septiembre & 120,7 & 770 & 92.918 \\
\hline Octubre & 99,1 & 820 & 81.250 \\
\hline Noviembre & 84,4 & 835 & 70.458 \\
\hline Diciembre & 88,3 & 660 & 58.274 \\
\hline Total & 100,1 & 8.983 & 899.107 \\
\hline
\end{tabular}

- Trastorno bipolar y códigos CIE 9 asociados: 100,1 días/año de promedio.

- Trastorno de ansiedad, depresivos y adaptativos y códigos CIE 9 asociados: 64,5 días/año de promedio.

Los datos comparativos entre los tres grupos y respecto al total de datos del INSS 2011 se muestran en las Tablas 4 y 5 (Figura 1).

\section{Comparativa por meses en estos procesos:}

- En esquizofrenia, no se observaron grandes diferencias en los distintos meses del año, excepto un pequeño repunte en el número de procesos en el mes de junio y una mayor duración media de los procesos en el mes de octubre.

- En trastorno bipolar no se observaron grandes diferencias en los diferentes meses del año, excepto una discreta disminución en el número de procesos en el mes de enero y en agosto y una mayor duración media de los procesos en el mes de septiembre.

- En trastornos ansiosos, depresivos y adaptativos no se observaron grandes diferencias en los diferentes meses del año, excepto una discreta 


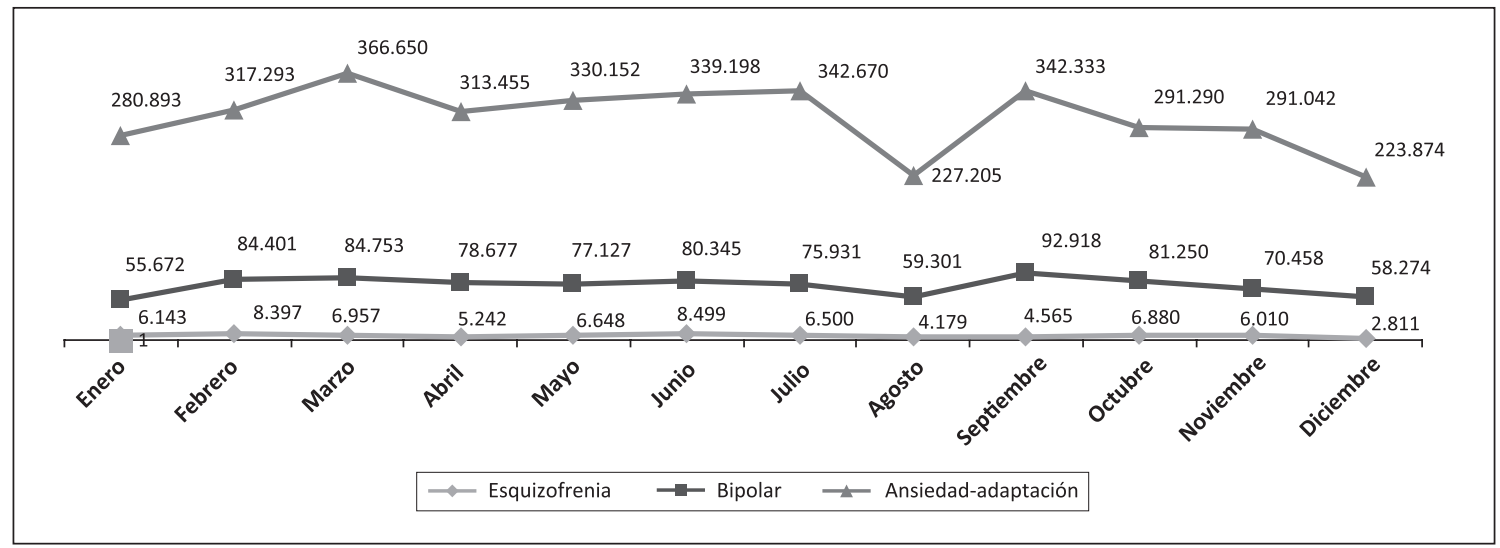

Figura 1. Días perdidos por IT en los tres procesos por meses: esquizofrenia, trastorno bipolar y asimilados y procesos ansiosodepresivos/adaptativos. Año 2011.

Tabla 4. Datos globales de jornadas perdidas por IT por meses en todas las patologías en 2011

\begin{tabular}{|lccc|}
\hline Mes del & Tipo de trastorno \\
año 2011 & Esquizofrenia & Bipolar & $\begin{array}{c}\text { Ansiedad, } \\
\text { depresión, } \\
\text { adaptación }\end{array}$ \\
Enero & 6.143 & 55.672 & 280.893 \\
Febrero & 8.397 & 84.401 & 317.293 \\
Marzo & 6.957 & 84.753 & 366.650 \\
\hline Abril & 5.242 & 78.677 & 313.455 \\
Mayo & 6.648 & 77.127 & 330.152 \\
Junio & 8.499 & 80.345 & 339.198 \\
Julio & 6.500 & 75.931 & 342.670 \\
\hline Agosto & 4.179 & 59.301 & 227.205 \\
\hline Septiembre & 4.565 & 92.918 & 342.333 \\
Octubre & 6.880 & 81.250 & 291.290 \\
\hline Noviembre & 6.010 & 70.458 & 291.042 \\
\hline Diciembre & 2.811 & 58.274 & 223.874 \\
\hline Total & 72.831 & 89.9107 & 3.666 .055 \\
\hline
\end{tabular}

disminución en el número de procesos en el mes de diciembre-enero y en agosto y duración media de los procesos prácticamente homogénea a lo largo de todo el año.

Comparativa de datos respecto al global de todas las patologías que han cursado con IT en España durante el 2011

Los trastornos esquizofrénicos han supuesto en España durante el 2011 el 0,053\% del total de procesos y $0,10 \%$ del total de días de IT. Los trastornos bipolares $0,55 \%$ del total de procesos y $1,26 \%$ del total de días de IT y el bloque formado por los trastornos ansioso, depresivos y adaptativos 3,48\% del total de procesos y $5,13 \%$ del total de días de IT, siempre considerando que los datos globales por todas las patologías incluyen procesos cuya alta se haya producido en el ejercicio 2011.

Tabla 5. Comparativo de cada una de las patologías respecto al total por todas las enfermedades durante 2011 por IT

\begin{tabular}{|lrccc|}
\hline & $\begin{array}{c}\text { Número de } \\
\text { procesos de IT }\end{array}$ & $\begin{array}{c}\text { Número de } \\
\text { días de IT }\end{array}$ & $\begin{array}{c}\text { Duración media de } \\
\text { los procesos de IT }\end{array}$ & $\begin{array}{c}\text { Porcentaje respecto al } \\
\text { total (datos INSS 2011) }\end{array}$ \\
$\begin{array}{l}\text { Total de procesos con } \\
\text { IT España-2011* }\end{array}$ & 1.631 .573 & 71.401 .033 & 43,76 & \\
\hline Esquizofrenia & 868 & 72.831 & 83,9 & $\begin{array}{l}0,053 \% \text { del total de procesos y } \\
0,10 \% \text { del total de días de IT }\end{array}$ \\
\hline Trastorno bipolar & 8.983 & 899.107 & 100,1 & $\begin{array}{l}0,55 \% \text { del total de procesos y } \\
1,26 \% \text { del total de días de IT }\end{array}$ \\
\hline $\begin{array}{l}\text { Ansiedad, depresión y } \\
\text { trastorno adaptativo }\end{array}$ & 56.795 & 3.666 .055 & 64,5 & $\begin{array}{l}3,48 \% \text { del total de procesos y } \\
5,13 \% \text { del total de días de IT }\end{array}$ \\
\hline
\end{tabular}

*Datos de procesos con alta se haya producido en el ejercicio 2011. 
Tabla 6. Coste aproximativo estimado por IT en todas las patologías en España durante 2011

\begin{tabular}{|c|c|c|c|c|c|}
\hline Proceso & $\begin{array}{l}\text { Esquizo- } \\
\text { frenia }\end{array}$ & T. Bipolar & $\begin{array}{l}\text { Ansiedad, } \\
\text { depresión, T. } \\
\text { de adaptación }\end{array}$ & $\begin{array}{l}\text { Datos globales } \\
\text { INSS** }\end{array}$ & $\begin{array}{l}\text { Porcentaje respecto al } \\
\text { total (datos INSS 2011) }\end{array}$ \\
\hline $\begin{array}{l}\text { Días perdidos } \\
\text { por IT }\end{array}$ & 72.831 & 899.107 & 3.666 .055 & 71.401 .033 & \multirow{2}{*}{$\begin{array}{l}\text { Esquizofrenia }=0,072 \% \\
\text { Trastorno bipolar }=0,89 \% \\
\text { Ansiedad, depresión y trastorno } \\
\text { adaptativo }=3,64 \%\end{array}$} \\
\hline $\begin{array}{l}\text { Coste } \\
\text { estimado* }\end{array}$ & $1.557 .126,78 €$ & $19.222 .907,66 €$ & $78.380 .255,9 €$ & $2.154 .355 .000,78 €$ & \\
\hline
\end{tabular}

${ }^{*}$ Coste día SMI = 21,38 euros al día. **Fuente: Indicadores INSS-2011. Ministerio de Empleo y Seguridad Social. Secretaria de Estado de la Seguridad Social.

Coste económico aproximativo de referencia a mínimos (asumiendo los sesgos mencionados anteriormente).

Los resultados ajustados al coste/día del SMI, se muestran en la Tabla 6.

El gasto indirecto relacionado con los días de IT a cargo del INSS de estas patologías durante el 2011 fue de aproximadamente $€ 99.160 .290,34$, lo que supone respecto al total del gasto por todas las patologías de 4,60\% (total gasto del INSS en IT durante el 2011= $€ 2.154 .355 .000,78$ ), siendo el mayor porcentaje de gasto el de los trastornos ansiosos, depresivos y procesos adaptativos con $3,64 \%$ del total $(€ 78.380 .255,9)$, seguido del trastorno bipolar con $0,89 \%(€ 19.222 .907,66)$, correspondiendo el menor porcentaje al trastorno esquizofrénico $0,072 \%$ del total $(€ 1.557 .126,78)$.

\section{Discusión}

Los resultados obtenidos muestran la superioridad en todos los parámetros de IT a cargo del INSS de los procesos ansiosos, depresivos y adaptativos, frente a los bipolares y esquizofrénicos, con escasas diferencias por meses en las tres patologías excepto un leve descenso en trastornos depresivos en agosto, si bien la duración media de los procesos es mayor en los trastornos bipolares y esquizofrénicos, aunque presentan un menor número de procesos anuales.

Los resultados de costes obtenidos han de ser valorados en su justa medida y como meramente orientativos, pues se toma como base un coste día mínimo, que corresponde al SMI ${ }^{14}$ y por ello se asumen múltiples sesgos, ya reseñados previamente y con cifras de gasto muy generales y en base a los datos de jornadas perdidas por IT de una institución de la seguridad social en España. La metodología para la estimación de costos es indirecta y probablemente insuficiente puesto que no se basa en los datos de las personas, diferencias existentes por sexos, bloques de edad, sector profesional de origen, etc. y sólo considera un componente del total de los costos por incapacidad temporal a cargo del INSS, sin que se tengan datos de la población de origen, ya que sólo incluye a los trabajadores a cargo de la seguridad social.

La patologías psiquiátricas estudiadas, han sido seleccionadas por los autores por considerarlas cualitativa y cuantitativamente importantes y con repercusión en costes indirectos asociados al gasto socio-laboral que implican las jornadas perdidas por IT en España, incluso teniendo en cuenta el hecho de que la mayoría de las personas con esquizofrenia o trastorno bipolar, están desempleadas o excluidas del mundo laboral y presentan importantes dificultades para acceder y mantenerse en puestos de trabajo remunerado ${ }^{15}$. No ocurre lo mismo con los procesos ansioso-depresivos que son compatibles con actividad laboral y suponen un alto número de jornadas perdidas por IT y, con ello, costes indirectos asociados.

Diversos autores han insistido en la necesidad de instaurar programas de rehabilitación laboral de apoyo a estos pacientes, pero sólo el $25 \%$ de los pacientes con esquizofrenia tienen un empleo remunerado y la mayoría a tiempo parcial ${ }^{16}$.

Los resultados obtenidos orientar hacia la instauración de actuaciones conjuntas a cargo, tanto de la medicina asistencial, como de la laboral y tendentes a reducir la repercusión de estos procesos mediante programas de control de la enfermedad y de integración del enfermo en el mundo del trabajo, sin que ello suponga un incremento en los costes de gestión de las empresas. Estudios similares en cuanto a costes han sido realizados por nuestro grupo en otras patologías como lumbalgia y patología de columna lumbar ${ }^{17}$ o cáncer de mama ${ }^{18}$, permitiendo cuantificar el coste de los procesos de una forma orientativa $y$ 
establecer comparativas entre algunas de las más prevalentes. Cualquier actuación conjunta por parte de todos los afectados que implique reducción en el número de procesos, en la duración de los mismos y en las jornadas perdidas tanto con cargo al empresario, como con cargo a las arcas públicas, en este caso a los fondos de la seguridad social española, supondrá un beneficio social y también económico que permitiría una optimización de los medios a nuestro alcance con implicaciones globales favorecedoras para el enfermo, la sociedad y las empresas e instituciones públicas, siendo para ello imprescindible la colaboración del Médico del Trabajo.

La legislación española en prevención de riesgos laborales nos da herramientas al respecto, siendo la más destacada en este caso el concepto de trabajador especialmente sensible, en el Art. 25 Ley $31 / 1995^{19}$, que recoge el espíritu de otras legislaciones similares en los distintos países europeos al amparo de la DIRECTIVA MARCO relativa a la aplicación de medidas para promover la mejora de la seguridad y de la salud de los trabajadores en el trabajo ${ }^{20}$.

\section{Referencias}

1. Clasificación Internacional de Enfermedades. (9.a revisión). Modificación Clínica. CIE-9-MC. Madrid: Ministerio de Sanidad y Consumo, 6a Edición. Enero 2008.

2. Calcedo Ordoñez A. Psiquiatría y ley. En: Tratado de Psiquiatría. Dirigido por Julio Vallejo Ruiloba y Carmen Leal Cerós. Ars Médica, Barcelona, 2005

3. Mingote Adán JC, del Pino Cuadrado P, Huidobro Á, Gutiérrez García D, de Miguel Peciña I, Gálvez Herrer M. El paciente que padece un trastorno psicótico en el trabajo: diagnóstico y tratamiento. Med segur trab [revista en la Internet]. 2007 sep [citado 2012 jun 16]; 53 (208): 29-51. Disponible en: http:// scielo.isciii.es/scielo.php?script=sci_arttext\&pid=S0465546X2007000300005\&lng=es. http://dx.doi.org/10.4321/ S0465-546X2007000300005.

4. Benavides FG. III health, social protection, labour relations, and sickness absence. Occup Environ Med 2006; 63: 228-9.

5. Vicente-Herrero MT, Terradillos García MJ, Capdevila García LM, Ramírez Iñiguez de la Torre MV, Aguilar Jiménez E, López-González AA. Minusvalía, discapacidad e incapacidad. Una revisión desde la legislación española Semergen. 2010; 36: 456-61.
6. Saha S, Chant D, Welham J, McGrath J. A Systematic Review of the Prevalence of Schizophrenia. PLoS Med (2005) 2 (5): e141. doi:10.1371/journal.pmed.0020141.

7. American Psychiatric Association. Guías Clínicas para el tratamiento de los trastornos psiquiátricos: Compendio 2004. Barcelona: Ars Medica; 2004. Disponible en htpp:// www.fisterra.com/guías2/bipolar.asp.

8. Walters MS, Kessler RC, et al. Prevalence, Severity, and Comorbidity of 12-Month DSM-IV Disorders in the National Comorbidity Survey Replication. Arch Gen Psychiatry 2005; 62: 617-27.

9. Ketter TA. Diagnostic features, prevalence, and impact of bipolar disorder. J Clin Psychiatry 2010; 71 (6): e14.

10. Laxman KE, Lovibond KS, Hassan MK. Impact of bipolar disorder in employed populations. Am J Manag Care 2008; 14 (11): 757-64.

11. Skokauskas N, Gallagher L. Psychosis, affective disorders and anxiety in autistic spectrum disorder: prevalence and nosological considerations. Psychopathology. 2010; 43 (1): 8-16. Epub 2009 nov 6.

12. Gustavsson A, Svensson M, Jacobi F, Allgulander C, Alonso J, Beghi E, et al; CDBE2010Study Group. Cost of disorders of the brain in Europe 2010. Eur Neuropsychopharmacol 2011; 21 (10): 718-79. Epub 2011 Sep 15.

13. American Psychiatric Association-DSM-5 Development. Descargable en: http://www.dsm5.org/Pages/Default. aspx

14. España. Real Decreto 1795/2010, de 30 de diciembre, por el que se fija el salario mínimo interprofesional para 2011. Boletín Oficial del Estado, núm. 318 de 31 de diciembre de 2010, páginas 109401 a 109403 . BOEA-2010-20150 Ministerio de Trabajo e Inmigración.

15. Crespo Hervás MD. Rehabilitación y psicoeducación en la esquizofrenia. Cap. 33. En: Las esquizofrenias. Sus hechos y valores clínicos y terapéuticos. Elserier, Masson, Madrid, 2007.

16. Chinchilla Moreno A. Desde lo conceptual esquizofrénico hasta su comprensión y clínica. Cap. 16. Las esquizofrenias. Sus hechos y valores clínicos y terapéuticos. Elserier, Masson, Madrid, 2007.

17. Vicente-Herrero MT, et al. Las enfermedades de la Columna Lumbar y su relación con el trabajo en España. Seguridad y Medio ambiente. Fundación MAPFRE. Año 32, no 126. segundo trimestre 2012.

18. Bandrés Moyá F, Delgado Bueno S, Vicente Herrero MT. Cáncer de mama, trabajo y Sociedad. Fundación Tejerina-Observatorio Europeo de patología de la Mama. ADEMAS comunicación gráfica SL. 2012.

19. Ley 31/1995 de 8 de noviembre, de Prevención de Riesgos Laborales. (BOE 10.11.1995).

20. Directiva 89/391/CEE del Consejo de 12.06.1989. 